\title{
RISE Microscopy: The Benefits of Correlative Microscopy for High Resolution Structural and Chemical Imaging of Biominerals
}

\author{
Ute Schmidt $^{1}$, M. Eder ${ }^{2}$, Wei Liu ${ }^{3}$, David Steinmetz ${ }^{1}$ and G. Wille ${ }^{4}$ \\ 1. WITec GmbH, Ulm, Germany. \\ 2. Carl Zeiss Microscopy GmbH, ZEISS Group, Oberkochen, Germany. \\ 3. WITec Instruments, Knoxville, TN. \\ 4. BRGM, French Geological Survey, Orleans, France.
}

RISE Microscopy is a novel correlative microscopy technique that combines confocal Raman Imaging and Scanning Electron (RISE) Microscopy within one integrated system. A Scanning Electron Microscope equipped with various accessories and detectors is a powerful tool for scientific inquiries that provides information on morphology, elemental composition and crystalline structure. Confocal Raman Imaging of the same sample area reveals the chemical composition as well as polymorphism, stress states and anisotropies [1-2].

Biominerals are heterogeneous materials which form complex inorganic-organic structures with unique and sought after properties. The superior mechanical properties, solubility and polymorphism of biominerals are achieved by a hierarchical construction employing organic components to regulate the precipitation of the inorganic phase. In this study we present structural and chemical results achieved via RISE microscopy on pearls and nacre, both valued as material for jewelry. Their regular form of mineralization is aragonite, an orthorhombic polymorph of calcium carbonate. Each bio-carbonate platelet is a poly-granular composite of aragonite nano-grains fixed by proteins. These platelets have a polygonal shape several hundreds of nanometers thick, which stack along the c-axis with chitin organic 'cement'. This layered structure produces the well-known pearlescence. Fig. 1a shows the SEM image of such polygonal shaped platelets acquired from an uncoated pearl surface using an HSBSE detector. Using this detector, a small contrast between individual platelets can also be visualized along with the shape of the platelets. The color-coded Raman image acquired from the same sample area is shown in Fig. 1b. The colors in the Raman image correspond to the colors of the Raman spectra presented in Fig. 1c. All three Raman spectra show the characteristic Raman bands of aragonite, however, the differences between these spectra is highlighted in the insert, representing the spectral area of the Ca-lattice vibrations. The brighter areas in the SEM image can be easily correlated with the red areas of the Raman image, indicating that there is an anisotropic arrangement of the platelets in the pearl (Fig. 1d). An intercalation of trace elements can be excluded because none of the Raman bands show a broadening or a shift.

A similar result was obtained from a polished section of nacre, consisting also of aragonite platelets. In this experiment, however, the stacking of the platelets was analyzed. Such an anisotropy can be found over several stacks along the c-axis in the nacre.

The example shown highlights the power of RISE Microscopy for the analysis of composite materials consisting of only one polymorph of calcium carbonate in terms of structural and anisotropic arrangement of platelets within pearlescent materials. In this contribution further examples will be presented, revealing the joy of multimodal correlative microscopy. 
References:

[1] J. Jiruše et al, J. Vac. Sci B 32 (2014).

[2] G. Wille et al, EMAS 2017 - 15th European Workshop and IUMAS-7 Meeting, Konstanz, Germany (2017).
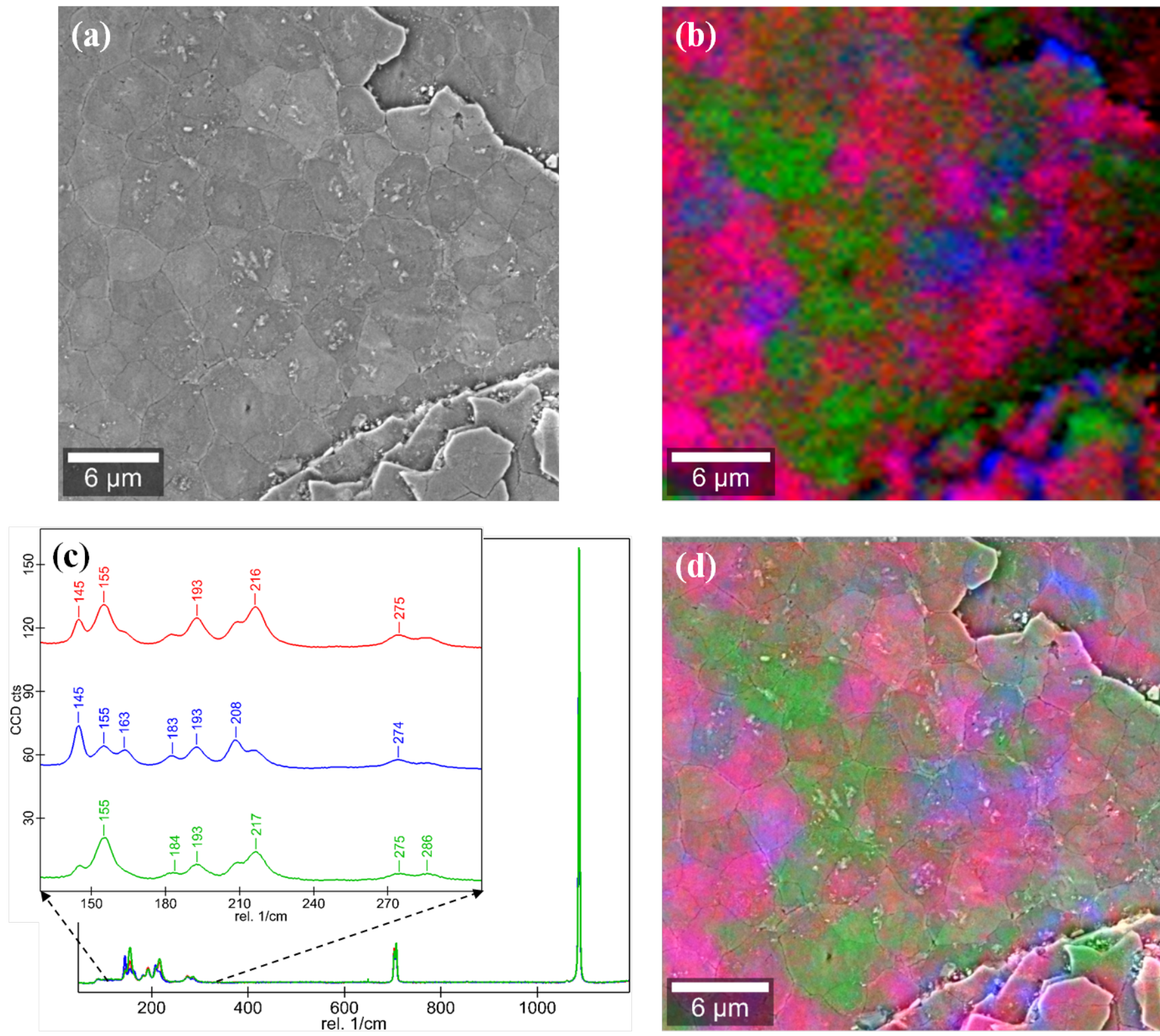

Figure 1. RISE microscopy study with a Sigma300-RISE of a pearl: (a) SEM image of the aragonite platelets acquired under low vacuum conditions with the HSBSE detector at $7 \mathrm{kV}$. (b) Color Raman image evaluated from a 2D-array of 120 x 120 complete Raman spectra (integration time: 0.2 s/spectrum, excitation laser: $532 \mathrm{~nm}, 50 \mathrm{~mW}$ ). (c) Raman spectra evaluated from the 2D spectral array. (d) RISE image of the analyzed sample area. 